\title{
Targeting the androgen receptor axis at the time of RP
}

\author{
Alan I. So, MD, FRCSC \\ Department of Urologic Sciences, University of British Columbia, Vancouver, BC, Canada
}

Cite as: So Al. Targeting the androgen receptor axis at the time of radical prostatectomy. Can Urol Assoc J 2021;15(8):280. http://dx.doi.org/10.5489/cuaj.7494

See related article on page 269

A Ithough radical prostatectomy (RP) may achieve excellent oncological control in most men with localized prostate cancer, biochemical recurrence is as high as $15-35 \%$ in large case series. ${ }^{1,2}$ Studies to improve outcomes after surgery have used neoadjuvant and adjuvant strategies applying androgen-directed therapy (ADT). The results of Nayak et al's meta-analysis in this issue of CUAJ re-confirms previously published data showing that ADT alone, neoadjuvantly or adjuvantly, does not improve overall survival (OS) in men undergoing $\mathrm{RP}^{3}$ and is discouraged in many guidelines. ${ }^{4,5}$ Hence, the additive benefits of adjuvant ADT seen with radiotherapy are not observed when ADT is combined with RP in localized disease.

The only patient population in which survival benefits have been observed in a randomized clinical trial with immediate ADT monotherapy post-surgery are those with defined metastatic disease; the ECOG 3886 study showed the potential benefit of lifelong ADT in men with $\mathrm{N}+$ disease post-RP. ${ }^{6}$ Nonetheless, issues of trial design (small patient population, delayed treatment in men in the control arm, and lack of prostate-specific antigen [PSA] screening in randomized patients) limits the applicability of this study in contemporary prostate cancer management.

The growing recognition of the side effects of even shortterm ADT, combined with the possible over-treatment in a significant proportion of patients undergoing RP, highlight the importance of identifying patients who are at high risk for disease recurrence post-prostatectomy and who will benefit most from neoadjuvant or adjuvant therapies. ${ }^{7}$ Although pathological features, such as margin positivity $(R+)$, as well as pT3a and pT3b disease, are most commonly used, novel molecular tests, such as Prolaris ${ }^{\circledR}$, Oncotype Dx ${ }^{\circledR}$, Decipher ${ }^{\circledR}$, and ProMark ${ }^{\circledR}$, may provide more precise identification of patients that require more aggressive, intensified approaches combined with RP. Use of these and forthcoming genomicbased tests will likely change how high-risk men undergoing $\mathrm{RP}$ are identified in future clinical trials and delineate those that require additional neoadjuvant or adjuvant therapies.

Future studies will also elucidate the role of novel agents that inhibit the androgen receptor pathway (ARPis) in men undergoing RP. There are many ongoing studies employing ARPis: neoadjuvant studies include using apalutamide and degarelix with or without apalutamide, and adjuvant studies include using darolutamide and apalutamide. Intuitively, these agents, which have more profound suppression of the androgen receptor axis, will have the greatest potential to positively influence outcomes post-RP.

Other unanswered clinical questions germane to the assessment of the value of ADT and novel ARPis include impact on patients' quality of life, optimal length of treatment, and benefit of combining ADT or ARPis with other systemic therapies. Since the androgen receptor axis is critical for prostate cancer progression, the use of agents targeting this pathway will be associated with any systemic therapy tested in men undergoing RP, either neoadjuvantly or adjuvantly. The results from Nayak et al show that when used alone, ADT prior to or after RP does not appear to significantly improve outcomes. However, as the authors suggest, more research to assess the value of targeting the androgen receptor axis before or after $\mathrm{RP}$, alone or with other systemic therapies, is warranted.

Competing interests: Dr. So has been an advisory board member for Abbvie, Amgen, Astellas, Bayer, Janssen, Ferring, and TerSera; and has participated in clinical trials supported by Astellas, Ferring, and Janssen.

\section{References}

1. Pound CR, Partin AW, Eisenberger MA, et al. Natural history of progression after PSA elevation following radical prostatectomy. JAMA 1999;281:1591-7. https://doi.org/10.1001/jama.281.17.1591

2. Stephenson AJ, Scardino PT, Eastham JA, et al. Preoperative nomogram predicting the 10-year probability of prostate cancer recurrence after radical prostatectomy. J Natl Cancer Inst 2006;98:715-7. https://doi.org/10.1093/inci/dij190

3. Nayak AL, Flaman AS, Mallick R, et al. Do androgen-directed therapies improve outcomes in prostate cancer patients undergoing radical prostatectomy? A systematic review and meta-analysis. Can Urol Assoc J 2021;15:269-79. http://dx.doi.org/10.5489/cuai.7041

4. Schaeffer E, Srinivas S, Antonarakis ES, et al. NCCN guidelines insights: Prostate cancer, version 1.2021. J Natl Compr Canc Netw 2021;19:134-43.

5. Fang D, Zhou L. Androgen deprivation therapy in nonmetastatic prostate cancer patients: Indications, treatment effects, and new predictive biomarkers. Asia Pac J Clin Oncol 2019;15:108-20. https://doi.org/10.1111/aico.13108

6. Messing EM, Manola J, Yao J, et al. Immediate versus deferred androgen deprivation treatment in patients with node-positive prostate cancer after radical prostatectomy and pelvic lymphadenectomy. Lancet Oncol 2006;7:472-9. https://doi.org/10.1016/S1470-2045(06)70700-8

7. Keating NL, $O^{\prime}$ Malley AJ, Smith MR. Diabetes and cardiovascular disease during androgen deprivation therapy for prostate cancer. J Clin Oncol 2006;24:4448-56. hrtps://doi.org/10.1200/JC0.2006.06.2497

Correspondence: Dr. Alan I. So, Department of Urologic Sciences, University of British Columbia, Vancouver, BC, Canada; alan.so@ubc.ca 